Article

\title{
Understanding Digital Health as Public Pedagogy: A Critical Framework
}

\section{Emma Rich ${ }^{1} *$ and Andy Miah ${ }^{2}$}

1 Department of Education, University of Bath, Bath, North East Somerset BA2 7AY, UK

2 Creative Futures Institute, School of Creative \& Cultural Industries, University of the West of Scotland, Paisley, Scotland, PA1 2BE, UK; E-Mails: andy.miah@uws.ac.uk; email@andymiah.net

* Author to whom correspondence should be addressed; E-Mail: E.Rich@bath.ac.uk; Tel: +44-(0)-122-538-6638.

Received: 22 April 2014; in revised form: 28 May 2014 / Accepted: 29 May 2014 /

Published: 10 June 2014

\begin{abstract}
This paper argues on behalf of a public pedagogy approach to developing a critical understanding of digital health technologies. It begins by appraising the hitherto polarised articulations of digital innovation as either techno-utopian or techno-dystopian, examining these expectations of technology and considering the tensions between them. It subsequently outlines how a public pedagogy approach can help mediate between these views, offering a more contextualised, socio-political perspective of mHealth. This approach teases out the nuances of digital health by engaging with the complexities of embodied learning. Furthermore, it urges caution against viewing these pedagogical forces as one of transference, or simple governance. To this end, we therefore contextualise our critique of digital health, within an attempt to reconstitute an understanding of public pedagogies of technology.
\end{abstract}

Keywords: public pedagogy; mobile health; mHealth; digital health; body; prosthetics; technology; learning

\section{Introduction}

In recent years, the surveillance of people's lives, bodies and health has been accentuated by the proliferation of digital systems and, in particular, the development of Internet-based technologies, which have significantly changed the way people engage with the data that surrounds their lives. The 
range of digital tools available is broad and a considerable amount of research has taken place to study how people negotiate their health in digital spaces, most apparent within the archives of the Journal of Medical Internet Research.

For many years, the focus of cybermedical encounters was on interactions that take place through websites and the wider impact of computer culture. Within this body of work, there was a tendency to engage with discourses of the utopian or dystopian consequences that may arise from an increasingly digital society. The Internet was considered to be a place that had the potential to change society in fundamental ways. In the case of the utopian expectations, early studies about the Internet wrote of how it would revolutionise society, breaking down social barriers and making the world a better place. Research focused on structure and inter-personal dimensions, which would free people from burdens associated with the physical world, such as the constraints of image and identity [1,2]. Such prospects led some researchers to advocate the alteration or elimination of bodies as a desirable future for humanity $[3,4]$. The obsolete body was thus seen as the manifestation of a techno-utopia.

In the context of health care, these expectations were reinforced by views that foresaw the capacity of digital technology to create a more cost-effective way of delivering health care. Moreover, the proliferation of digital technologies across all sectors of society would lead to an increased utilization of and reliance upon digital solutions within the healthcare sector. Emerging out of an era of telemedicine and, later, discourses around "cybermedicine" and mobile health [5], the appeal of digital solutions occurred against a backdrop of welfare cuts, rising health care concerns about the global increase in "lifestyle" diseases such as obesity. In this sense, digital health — as a focus for policy investments - flourishes as a result of its capacity to generate greater efficiencies within an already overburdened system. Integral to this utopian discourse on cybermedicine's capacity to revolutionize healthcare, a further discourse has developed that focuses on how digital technology could radically alter the quality of provision and better reinforce some of the ethical aspirations of medicine. For instance, researchers wrote about how health care could promote patient autonomy and professional accountability [6,7]. In this sense, digital health was not just a way of delivering more efficient care, but better quality care as well.

In contrast, among the public discourse on this brave new virtual world were anxieties about how it would lead to more disenfranchised, lonely individuals, with diminished social skills and, potentially, dispositions that would be bordering on clinically alarming, addiction to cyberspace being a commonly expressed concern. Such dystopian views were articulated in relation to video game culture, framed by a similar kind of moral panic that has surrounded other new media forms, such as certain genres of popular music, or film. Thus, the Internet was seen as a place where bad things happen to otherwise good people. These views have been re-articulated with each new innovation online, from social media to wearable technology [8,9]. For instance, neuroscientist Susan Greenfield is frequently given space in the media to warn people of virtual worlds, claiming that too much time online can even be detrimental to processes within the brain [10].

As web studies emerged more formally in the late 1990s, and as more rigorous methodological approaches developed for analysing what takes place online [11], less attention was given to the long term transformative dimensions of the Internet, and more focus was placed on what was actually taking place online. As a result, the research community of digital studies scholars fragmented very early on in the development of this new thematic area and this fracturing makes it difficult to assert 
that there was a single techno-utopian vision of cyberspace that was envisioned for health care. Equally, policy debates focused more on the instrumental benefits of telemedicine and their parameters were far narrower than imagining anything as grand as utopia. Indeed, too much digital health was seen as antithetical to utopia, at least in terms of governance. The rise and fall of Google Health evidences the limitations of digital health interventions aimed at transferring responsibility wholly to the individual away from the state.

In this context, our initial critical reaction to discourses on digital health is to deny that there was ever a sufficiently wide perspective on the prospects of digital health to warrant its being described as a techno-utopian project. Instead, there were instrumental aspirations around promoting digital health, which were about governance and, to some extent, ethics. However, there was no wider moral framework in which these aspirations for health care were situated. Thus, if a utopia-in the commonly employed sense of the term-may be used to describe a state of perfection, then the instrumental digital health discourse was considerably lacking, since it failed to attend to some of the complex consequences of providing health care at a distance.

In sum, looking back over the early years of web studies reveals a degree of mythology about the Internet's potential, whereby the few futurists and scholars who occupied this territory became disproportionately influential at shaping the discourse about this subject area. The likes of Howard Rheingold [12] and even Sherry Turkle's [13] early work, were, on balance, minor influences in the trajectory of social scientific studies of digital health, but their libertarian claims are probably best ignored when attempting to engage with the development of the discipline's history. It is reasonable to be ambivalent about this state of affairs, since much of the instrumental research surrounding digital health - typified by the articles published in the Journal of Medical Internet Research - would benefit from greater scrutiny from a cultural theory lens. Indeed, a lot has changed since these early years of theorising the web and this deserves some critical reflection, so as to understand what interpretations are appropriate today to arrive at a more critical view.

\section{The Mobile Web and App Culture}

One clear insight from this early period is that it is important for researchers to consider what still needs to be investigated in digital health studies, but perhaps more crucially, what critical theoretical lens is most appropriate to address the complexity of what takes place online. We address this by focusing on the trend towards utilizing mobile devices to navigate the Internet. Mobile devices are playing an increasing role in the digital economy, experiencing more growth than computer sales worldwide. Furthermore, the number of mobile devices that exist in the world already exceeds the world's population. Moreover, it has become apparent recently that mobile web usage is in decline and, instead, a growth in mobile app usage has been registered [14]. This means that people are using mobile browsers and websites less, choosing instead to access content directly through mobile application stores, such as the Google Play store and Apple App Store. This is a compelling reason to study how people engage with their health via mobile devices.

Yet, it also appears that "mHealth" [5] is quickly becoming a core mechanism through which people become complicit in engendering systems of surveillance that can be framed by commercial or political interests. Unlike web browsers, which are relatively transparent in how they capture and 
monitor a user's data, users of mobile apps may have little understanding of how the data that is captured about their health is utilized. However, such data can have dramatic impacts on various industries who will happily pay for access to such information to further their goals, whether these are governmental or commercial. The increasingly ubiquitous presence of mobile devices ensures their place as a core driver of health engagement. The global significance of this cannot be underestimated, as mHealth is capable of functioning in environments where there is no cabled broadband infrastructure, which means it is more suited to use within nations that have limited infrastructures. The importance of the mobile device market and, in particular, 3G and 4G infrastructure is made apparent in the recent investments by both Google and Facebook in airborne Internet provision. Thus, studying mobile culture in the context of health is of particular value, as it resonates with the direction of travel in global communications in a way that more effectively alleviates the digital divide.

We also narrow our analytical lens even further by looking specifically at mobile health apps that relate to physical activity and lifestyle. Such apps, which allow users to track their exercise behaviour, body weight, and food consumption, represent a significant proportion of the health app market, making their communities a critical mass of interest. Indeed, exercise, body weight and dieting apps are amongst the most popular downloaded health apps [15]. Moreover, 80\% of the population in Europe has carried out a health-related search on the Internet [16] and mobile phone subscriptions worldwide reached nearly 7 billion in 2013 [17]. In this context, the present paper focuses on the utilization of self-tracking mobile technologies associated with physical activity lifestyle and health promotion.

Changes in public health promotion focused on "lifestyle" have been brought about by rapid developments in digital health technologies [18]. In recent years, healthcare and health promotion has operated through "e-scaped medicine" [19] no longer confined to clinical institutions but, instead, delivered through "diffuse and fragmented" networks of locations. This has led to a proliferation of research that explores how best to utilize digital systems to predict, diagnose, treat, and monitor health. At the same time, it has created patients who are much more inclined to use technology to monitor their health and, thus, be more complicit in their own surveillance, albeit within platforms where data that is consumed is under the watchful gaze of the platform owners and unspecified third parties who can purchase their data for their own goals. Indeed, one of the challenges with the growth of mobile health apps is the difficultly we have in understanding the information chain where our own agency becomes a commodity for use by other organizations.

In others words, while greater access to technology may have narrowed the digital divide, the rapid transformation of digital platforms - if only from Web 1.0 to Web 2.0 architecture - has widened the digital literacy divide. These circumstances compel researchers to think critically about the locations in which a public understanding of digital culture develops, beyond formal education systems. This is particularly important in the context of health, since being able to negotiate the digital terrain effectively can determine one's capacity to experience greater autonomy or to derive greater insights into one's health. Yet, one may look precisely to how education has also changed as a result of the Internet era and bringing together these two dimensions - health and education - is a helpful way of developing a critical approach to mHealth. Indeed, education has expanded beyond its conventional realms over the last decade. The trajectory towards lifelong learning, and even such programmes as "one laptop per child" or Sugata Mitra's "hole in the wall" programme, speak to the wider spaces in which innovative educational practice is delivered through technology and often within a public 
context. Other examples, such as MOOCs and qualification "badges" each further articulate how traditional education systems are developing more publicly facing programmes.

\section{A Public Pedagogy Approach to mHealth}

This shift has been accompanied by a groundswell of scholarship that draws on the theoretical construct of "public pedagogy". Whilst the emergence of the field of public pedagogy has presented possibilities for new approaches to understanding education, it consists of varied perspectives. In their comprehensive mapping of the field and review of literature spanning 1894-2010, Sandlin O'Malley and Budrick [20] identify a lack of definitional theoretical clarity in the way in which public pedagogy has been conceptualised and applied across a broad set of literature, from the role of school in preparing young people for citizenship through to the public pedagogies of dominant discourses. Approaches to public pedagogy have been informed by a broad range of literature including curriculum studies, adult learning, lifelong learning, critical pedagogy, feminist studies.

As an analytical device, public pedagogy is located at the intersection of numerous disciplines, including but not limited to pedagogy, sociology, and cultural studies. On this basis, it offers exciting potential for critical explorations of the relationships between the educative force of a range of cultural sites in people's lives and engagement with physical practices, corporeality and subjectivities. Inquiries into understanding how and what people are learning about the (in)active body through these emerging technologies aligns with a Physical Cultural Studies approach which David Andrews [21] (p. 45) defines as a:

"Synthesis of empirical, theoretical and methodological influences (drawn from, among other sources, the sociology and history of sport and physical activity, the sociology of the body, and cultural studies) that are focused on the critical analysis of active bodies and specifically the manner in which they become organized and represented, and experienced in relation to the operations of social power."

A growing body of influential work has examined the impact of new media on the organisation, representation and experiences of the body, operating within these various physical cultures Andrews describes above. Yet, as Freishtat and Sandlin [22] observe, there has been a limited body of work exploring the public pedagogies of technologically mediated spaces. This reveals a lack of explicit focus on the pedagogical processes operating in the digital environments described above. Drawing on a range of theoretical perspectives, questions have emerged as to how far health professions and individual users of technologies are prepared to incorporate these kinds of technological applications into their lives and what kinds of ethical issues might arise. Their legitimacy and desirability has been the focus of a number of important contributions to this debate across a range of disciplines (philosophy, surveillance studies, social sciences, cyberstudies, new media studies). The relationships between these technologies and their users have been explored through various theoretical perspectives of surveillance and governance, but these have not framed the relationship as one of learning or pedagogy. In attempting to contribute to this developing theoretical discussion, we approach these issues from a different perspective, endeavouring to make clear a justification for a critical engagement with mobile health technologies from a public pedagogy approach. Thus, we wish to reposition a 
critical perspective on digital health and suggest that an effective way of doing so is through the lens of public pedagogy. We do not argue that this approach should replace others, but that it offers a complementary approach within a multidisciplinary critical perspective that is capable of synthesising a range of critical priorities in how we analyse digital health interactions. As Hickey-Moody and colleagues [23] argue, public pedagogy approaches function as "bridges or rather, as multiple crossing points, between the fields of education, sociology and cultural studies".

Each of these transformations to understanding education and mHealth speak to a wider opening up of what were previously closed practices - health and education. What took place in each of these areas of society was locked behind institutional systems, such as schools, universities, doctor's surgeries and hospitals. In the era of mobile health, these encounters with health and education have become more public facing, social experiences. Furthermore, there is a trend towards greater visibility in digital spaces, a theme made evident in the recent popularity of "selfies"-self portrait photos taken on mobile devices. Indeed, a helpful example in the crossover between health issues and digital visibility is the recent "dare to bare" campaign, which saw women sharing selfies online without wearing makeup in order to show solidarity and support for cancer sufferers. Various commentaries have emerged around this grass roots phenomenon, but what seems salient to our inquiry is the way in which self-mediation interfaces with health and the manner in which somebody's public self is a vehicle for health communication and education. Thus, creating a \#daretobare selfie photograph may be considered a political act, as it engages people with discussions about authenticity, identity, solidarity, health and self hood, while also forces us to think about representation and individuality set against a proliferation of celebritised, photoshopped images which bear little resemblance to real people. Individuals who chose to 'dare to bare' were exercising their empowered, socially situated selves and, in so doing, were actively raising awareness about the need for cancer research, if not as individuals, then as a collective.

Explaining the trend towards greater visibility in digital space requires a range of conceptual apparatus, but Ruckenstein [24] (p. 68) gets close to what is at stake when discussing how "smart phones and tracking devices" have created a "field of personal analytics" and "self-monitoring practices". Thus, we steer away here from concluding that either digital technology is taking us towards a utopia where its users feel necessarily empowered or better off, or that digital media will ultimately enslave us to some dystopian nightmare scenario. Rather, it is useful to contextualise this distinction between perspectives that celebrate the value of digital technology and those that adopt a more worrisome approach. The rise of mobile health apps does not simply respond to a vision of health, but can also be considered characteristic of a "confessional society" [25]. To this end, we ask what theoretical frameworks might aid us in yielding more complex and nuanced understandings of surveillance within these contexts and help mediate between the above dichotomy. By drawing from, and building on, the contributions of an emerging field of public pedagogy scholarship, it is possible to ask different, perhaps deeper, questions about individuals' engagement with digital health technologies.

In advancing a public pedagogy approach to theorising digital health, it is necessary to recognise how technology is inextricable from the manner in which people learn about health. Furthermore, these apparatus dictate conditions of self-tracking, collection of data, and monitoring, which have a bearing on what and how people learn about their bodies and health. Yet, there is no singular approach to public pedagogy and so it is crucial to first clarify the analytical approaches they offer in critiquing 
digital health. Thus, we draw from the work of Jake Burdick and colleagues in "problematizing public pedagogy" [26] and we examine the utility of its current application and appropriation, while endeavouring to build on and develop this body of work so that it develops a more critical perspective of digital health.

Public pedagogy scholarship is flourishing at a time when the distinction between public and private spaces has been significantly complicated and confronted by our emerging digitality. These conditions frustrate some of the established narratives around public pedagogy scholarship. Thus, we are cautious to claim that these pedagogical forces lead to transference, or simple forms of governance. Instead, we seek to understand: "how these educational sites and practices actually work to teach the public and how the intended educational meanings of public pedagogies are internalised, reconfigured and mobilized by public citizens" [20].

Given the diversity of approaches to public pedagogy, what should be meant when invoking this concept to make a case for a more critical perspective of digital health? Whilst public pedagogy is a contested term, there is some general consensus within the field that it comprises a focus on the kind of learning that takes place outside of formal schooling. Thus, in developing an account of digital health as pedagogical, it is necessary to start with a vision of education that recognises how learning - albeit about one's body and health in this case - can occur in sites and contexts beyond formal schooling. This is precisely the approach that has characterised public pedagogy in recent years. A number of notable theorists [27-32] have drawn attention to contemporary sites of learning beyond the boundaries of formalized education sites, forming what has been announced as the field of "public pedagogy". Attention has therefore been recast in educational research towards processes of education that take place across a variety of sites, including museums, zoos, libraries, media, popular culture, commercial spaces, grassroots activists movements, and more recently through the work of "public intellectuals" (see $[20,32])$. In this paper, we extend this also to digital platforms, such as social media environments, which are being positioned as technologically mediated pedagogical spaces [22]. Yet, as Freishtat and Sandlin [22] (p. 505) go on to suggest "there is a significant gap in the literature inquiring into the emergence of technologically mediated spaces as locations of cultural production, education, and learning among youth." Furthermore, in line with the critiques by Glen Savage [33], the multiplicity of public pedagogy approaches raises questions about what counts as "pedagogical" phenomena. In digital health environments, this may be especially difficult to discern, since many of them may not be recognisable as "learning" environments.

Nevertheless, it is reasonable to acknowledge that a necessary, if not sufficient, condition of public pedagogy's utility lies in its focus on understanding education, as it occurs in sites beyond formal institutions. Underpinned by theoretical contributions from cultural studies, feminism, pedagogy, and other disciplinary perspectives, the focus of public pedagogy has been on more resistant forms of critical pedagogy, and on identifying dominant discourses, drawing attention to the educational force of popular culture [34]. However, recognising public pedagogy as a distinct field remains somewhat premature, as its conceptual trajectory is still being formed. Hickey et al. [23] propose the term "pedagogy writ large" to capture a general set of theoretical conceptualizations "public pedagogy" and "cultural pedagogy", which frame pedagogy in this broader sense.

Therefore, a perspective of digital health arising out of public pedagogy approaches must be mindful of the tendency for scholars to cite the "term without adequately explicating its meaning, 
context or location within differing articulations of the construct." [26] (p. 3). In doing so, whilst we advocate the need for an educational framing of digital health, we also consider the work of Burdick, Sandlin and O'Malley [26] and Savage [33] to be essential, when extending and problematizing notions of public pedagogy in the context of mHealth. At such a juncture, Budick et al. [26] (p. 3) offer an instructive trajectory for public pedagogy, based on an architecture of three key areas; "framing, exploring the problematics of public pedagogy's definition, the organization and historicity; studying, emphasizing the ways in which our research simultaneously illuminates and obfuscates the object of inquiry; and enacting, taking up the ways in which we view and engage with our own pedagogical acts outside of institutional spaces." This provides a useful architecture through which to outline a public pedagogy approach to digital health, whilst also calling into question some of the tensions inherent within these approaches. By drawing on their framework, we simultaneously apply public pedagogy to our critique of mHealth, while also exploring the problematics of its current definition (framing), and considering how this obfuscates certain aspects of learning through technology (studying).

\section{Governmentality and the Public Pedagogies of "Nag Technologies"}

If public pedagogy is characterised by how learning takes place outside of formal educational structures, then clearly a prominent feature of where such learning takes place is within those informational spaces that surround every day life, notably digital spaces. To this end, a further characteristic of public pedagogy may be the conditions of such experience, which, we recognise, has to do with the way people negotiate their public persona and the manner in which they are complicit in self-surveillance. After all, the promotion of healthy behaviours through mobile apps has intensified processes of surveillance and regulation of people's everyday lives, raising a number of questions about their applications. Such devices as iPads, Fitbit wristbands, patches, or GPS equipment, require users to collect and log data, which builds a profile of their lifestyle, including wear they have walked, how fast they walked, and what they did along the way. Indeed, part of the appeal of these tools is that they gamify [35] the experience of monitoring health, introducing playful dimensions that encourage user participation. This may include documenting exercise habits, diet, weight with reward systems, motivational tools, and all of this data builds a sense of the "quantified self" [5,36], while also linking this publicly through social media. Products such as the Nike+ fuelband (a wristband designed to monitor and measure "whole body movement") is a good example of this, since it also has a built in capacity to share achievements with friends. Thus, the platform integrates a public dimension predicated on the user's interest to share what they have done.

Users of these technologies are complicit in shaping the culture of self-surveillance that underpins digital societies and the degree to which people share what they do may speak to a willingness to be subject to an outsider's gaze upon their bodies. Yet, the legitimacy and desirability of these technologies has recently occupied public attention, with recent news media documenting the rise of what has been termed "nag technology"- that is, technology which persistently nags its user to be active and live a healthy lifestyle. The cultural and regulative connotations of this term are clear to see. The public pedagogic focus here is on the "dominant cultural discourses" within "popular culture and everyday life" [20], which compel people towards healthy behaviours. In other words, users are asked 
to learn how to look after themselves via the disciplining regularity of the device's presence and regular notifications to maintain their good behaviour. According to Sandlin et al. [20], current approaches within public pedagogy draw upon popular pedagogies to, "to link cultural and media artifacts to processes of social domination". Perhaps the most well-known theorist to have contributed to this work is Henry Giroux [27,37]. Giroux's work on popular culture and pedagogy and, particularly, "corporate public pedagogy" as a "a powerful ensemble of ideological and institutional forces whose aim is to produce competitive, self-interested individuals vying for their own material and ideological gain" [38] is particularly informative here. Furthermore, Giroux has been crucial in fashioning contemporary understandings of the educational force of such popular cultural sites as film, television, and the Internet.

Public pedagogy work of this nature draws on principles consistent with a focus on the dominant discourses promoted through educational sites, and the ways in which popular culture is utilised as a form of governmentality. From this perspective, dominant discourses of digital health are inextricably tied to the neoliberal discourses flowing through these digital technologies, demanding the neoliberal subject capable of acquiring information, monitoring and adjusting their bodies/lifestyles in relation to this information. Imbued with the negative implications of these technologies, which are partly consistent with the dystopian perspectives on digitality described earlier, it calls into question the narratives of empowerment that advocates of digital health would have us believe are the outcomes of a digital life.

When endeavouring to make sense of these shifts, Lupton [5] observes that the rapid growth and popularity of "mHealth" reflects the trend towards self-tracking as a way of managing one's lifestyle in line with the logic of a growing movement known as "the quantified self", where one collects and charts data, shares it and gathers real time feedback about it, which is then used to take better "care of the self" [39] and modify one's behaviour. This may involve sharing how far one has run and receiving encouragement from friends, which then incentivises the user to continue, or making weight loss targets. From this interpretation, one can critically explore how these digital devices act as pedagogical devices through certain pedagogical frames, such as the quantification of self, the confessional, surveillance, and the importance of self-evaluation. Mobile health technologies, particularly those associated with health promotion, exhibit particular pedagogic processes in the management of bodies. The transparency of these lessons provides a critical framework through which to examine the regulative force alluded to in this populist terming of digital health. Thus, mobile apps and social media sites are some of many examples of media texts or artifacts that warrant analysis as vehicles of public pedagogy.

With their accompanying processes of surveillance and evaluation, these technologies imply expectations of control, which are to be learned and reproduced by users. In this fashion, the appeal and explanatory potential of this conceptualisation lies in its analytical focus on the connection between popular culture and neoliberalism in explicating its educative force in the management of bodies through new digital health platforms. Pedagogically, this represents a shift from learning digital norms of techniques of the body [40] — of users knowledge of "how to use their bodies" - towards a focus on learning how to use information "about their bodies" [40] (p. 70). A good example of this is a mobile app that required users to make necessary plastic surgical interventions to an avatar to make her beautiful. As one tech magazine writes: "The game...rated for children 9 and older...walked players 
through the graphic steps of liposuction that must be performed on an "unfortunate girl" to make her "slim and beautiful" [41]. Compelled by a Twitter protest campaign going under the hashtag \#EverydaySexism, the app was eventually removed from the App store.

Such an example raises questions about how, increasingly, younger people engage with these technologies as pedagogical devices through which they learn to recognise themselves and/or others as good, healthy, active and/or having desirable bodies in the pursuit of "healthiness". Framed through the lens of public pedagogy, young people use these participatory technologies to learn not only about "health" activities, but also of what and whose bodies may be valued, given status, and how to meet the expectations of particular body pedagogies [42]. In the plastic surgery app, fatness is depicted as "matter out of place" [43] to be removed through processes of extreme modification. The regulation of and management of bodies in these mobile apps and social media environments corresponds with an idealised version of acceptable bodies and the abjection of others' bodies. In adhering to the corporeal order and normalisation of thinness, users are exposed to particular body pedagogies. Moreover, these applications depict particular bodies and subjects as marginal, different or even problematic and abject. Through game playing scenarios, the removal of adiposity through surgery reinforces the idea that "if embodied subjects fail to conform or perform to bodily norms, they stand to be classified as expendable waste; a waste that must be purified or hidden if society is to be maintained as a realm of order and productivity." [44] (p. 3). In the plastic surgery app, bodies that are overweight are therefore positioned negatively as abnormal and in need of modification, in contrast with the embodied subjects that emerge after surgery.

The educational function of these apps is to normalise the fight against fatness or obesity, which, in turn, may influence and condition its users. Therefore, these digital spaces emerge as important pedagogical devices, as they become sites through which users learn about their own and others bodies and "add to an understanding of how a curriculum of enculturation occurs within the social context of networked technologies" [22] (p. 509). Similarly, in the context of health promotion apps that monitor and regulate body data, Lupton focuses on "the kinds of meanings and the representation of the ideal subject that are related to the use of these technologies in the interests of promoting health" [5] (p. 232).

While it is apparent that one should question the legitimacy of the learning that takes place here, a deeper, more critical reading of the phenomenon relates to the pursuit of sharing the experience. Thus, what distinguishes public pedagogy in a digital environment is that the learning does not simply end with the user's experience, but that these "lessons" are then pushed out to wider communities. The consequences of such sharing also require exploration. Beer and Burrows [45] argue that "web cultures are defined by the consumption of the mundane", particularly through social media sites where a range of personal information about the user's everyday life is often made readily available. Whilst the criticisms of this may vary, it may be useful to focus on the explanatory potential of the "confessional society" [25] that such sharing describes, whereby there is an accompanying obligation to live private lives publicly.

These conditions are inextricable from the rise of the Web 2.0 ethos and the structural principles that govern these practices. Through a "culture of connectivity" [46], personal lives are organized around the retrieval and public display of data about a diverse range of aspects of one's body and lifestyle. In the process of collecting, visualising, sharing and monitoring such data on one's body in a public space, users learn about the body in terms of appropriate forms of maintenance, development 
and repair [47]. The blurring of health and these inter-personal communications involves a process of self-presentation online, which is achieved by sharing images and text. Yet, the public sharing of information and images about one's body and health, raises questions about new, potential vulnerabilities, such as online bullying, which has been identified as a particularly significant risk to children using the Internet in Europe [48]. It is thus particularly important, to consider the interface between learning via public pedagogy and more formal educational learning, perhaps making social media education as crucial as religious, drugs, sex, financial or pastoral education within a national curriculum.

So, the critical approach to public pedagogy espoused here, focused on interrogating broad dominant discourses, helps us to identify the neoliberal educational forces associated with consumerism, where commercial platforms introduce "new modes of surveillance" [46]. Certainly, this work provides central tenets for us to scrutinize technology from a critical perspective, assisting us in formulating an understanding of the political, social and cultural norms that shape citizenship through public pedagogy encounters. This is particularly important in the case of commercial health apps, which contribute to powerful discourses of the body in the context of consumerism that saturates most online environments [22] (p. 507). However, such interpretations of mHealth might be indicative of what Savage [33] terms the "enveloping negativity", which has plagued public pedagogy.

\section{Relationalities of Digital Health Pedagogies}

Thus far, we have recognised how public pedagogies of digital health must be located within perspectives that Sandlin et al. [20] define as propagating "dominant discourses" and reinforcing the "popular culture and everyday life". From this understanding, we have highlighted the tendency to polarise perspectives that may lead us to characterise digital health as wholly serving a surveillance society, when it does not. Rather, surveillance takes on a new meaning in a digital age, given the range of complicity that it implies by sharing one's "quantified self". Nevertheless, such perspectives offer important contributions to, for example, examining "the implicit curriculum of technologically mediated spaces and their public pedagogy" [22] (p. 507). Thus, despite the absence of explicit learning goals within such informal spaces, they nevertheless foster certain kinds of learning.

Whilst Giroux's work has been influential in recent applications of scholarship claiming to focus on public pedagogy, we must be cautious of appropriating such ideas in a vein that diminishes our capacity to understand the complexities of "cultures of connectivity" [46]. In this vein, we posit that, alongside the focus on the "content" and curriculum of digital health pedagogies, one must also consider the pedagogical relations that frame people's experiences of health within digital spaces. Thus, we now turn our attention to the "relational aspects of inhabiting a place and how these shape subjective experience with the kind of relational encounter that may occur" [49] (p. 57).

Our interpretation of public pedagogy as occurring within informal, digital, social spaces, captures the pedagogical features of technologically mediated health sites, whose form may be adequately encapsulated within the term "biopedagogy" [50]. Through this lens, we are able to make visible the discursive and educational forces, which evoke self-management techniques and neoliberal practices of the self. However, a focus only on the meanings found within the sites of digital health, and accompanying neoliberal modes of regulation, would be antithetical to our aspirations to better 
understand the complex pedagogical processes of these apps from a critical perspective. This may also leave a number of critical and complex questions unanswered, such as developing an understanding of the supportive role played by the network within these spaces, irrespective of what is learned formally about health. Perspectives characterised by visions of techno-dystopia, therefore, may be troubling in the sense that there is a danger of latent assumptions of docility present within their readings of technology. As Walkerdine [51] (p. 201) suggests "a relation between the effectivity of biopower and the subject working on the self, or resisting, is too simplistic...health works in complex ways to produce subjectivity and that we cannot just read subjectivity off from biopolitical modes of regulation". To do so, would be to invoke a techno-dystopian reading of these modes of regulation.

Whilst users might all experience the discursive organisation of their bodies through health apps, the public pedagogies we explore here may help us to understand how users experience this in relation to different "regimes of meaning" [52] which are formed relationally. In this sense, we may extend these pedagogical notions to consider the different relations of affect circulating through the intersecting publics that emerge online. In other words, whilst digital health technologies are the site of regulative discourses and practices, the adoption of these practices is not simple, nor can it be assumed in the broader readings of public pedagogy. As Ruckenstein observes, "the ways in which people confront and engage with visualized personal data are as significant as the technology itself” [24] (p. 69). But more than this, the way people experience discursive relations within these technologies is in relation to the circulation of affect through corporeal and prostheticised bodies. At the same time, there are critiques that broad visions of public pedagogy have been plagued by an "enveloping negativity" [33] through the focus on social dominance. Against this background, the call by Burdick et al. [26] to consider what our public pedagogy approach "obfuscates and illuminates" is even more salient in the context of understanding embodied learning through digital health. In other words, a focus on the biopolitical may help to define, but also limit, the reach of conceptualisations of embodied learning and the complexities of the body in digital spaces.

It is not that a focus on dominant discourses fails to provide valuable insight, but rather it we need be caution of focusing only the content of pedagogy rather than its relational derivation. Savage [33] warns against such "totalizing and mythologizing" approaches to public pedagogy, which become all encompassing and through which "popular public pedagogies, therefore, are reduced to little more than mechanisms for exercising ideological domination" [53] (p. 85). Our understanding of connectedness of bodies with technologies and other bodies is diminished as a result of this narrow approach and the potential for understanding the complexities of resistance, negotiation and contradiction may be obfuscated. In this sense, our critique extends the conceptual apparatus we bring to public pedagogy to make sense of what happens in mHealth experiences.

To recap, our approach to understanding mHealth rephrases the question of pedagogy in the context of relationalities. We argue that, rather than focusing only on the politics of digital health, or on the meanings it produces, we must consider the "complex, moving webs of interrelationalities" [30]. The importance of this relational approach is further evidenced by the reliance within public pedagogy on an increasingly unsustainable distinction between public and private [53], which is blurred by digitality. Whilst the commercial intrusion of media has been recognised elsewhere, web 2.0 technologies further compromise what may be understood as "private" matters. This presents a number of risks that are broader than those which are typically associated with life online and to the 
(re)framing of pedagogical address, as often occurring in unpredictable ways. Understanding how people learn to recognize themselves and/or others 'as good, health, active desirable bodies in the pursuit of "healthiness" within these environments means avoiding a simple distinction between "virtual" bodies and "offline worlds" or between public and private spaces.

As Glen Savage observes, this recognition reveals the complexities that arise from intersecting publics, when conceptualising of public pedagogies of the body [53]. Thus, our task in developing a critical perspective on mHealth is to understand and identify the extent to which pedagogical forces extend and "link people together as a public" [53] (p. 80) or as a learning community. Freishtat and Sandlin [22] (p. 505) suggest that social media can be understood as producing a habitus, such that a users "experiences with technological culture influence the ways in which they will interact with technology". Moreover, an individual's engagement with their body occurs dialogically in relation to family, gender and socio-cultural and local contexts, as they engage with these digital spaces. So, on the one hand, these apps and their accompanying imagery of the desirable body, engage people with particular body pedagogies, though they are also re-imagined through local meanings and contexts. This reading of digitality frustrates conceptions of technology that might position it as ultimately surveillant and disempowering in an absolute sense, rendering people devoid of critical agency. After all, such perspectives behove us to ask how complex relationalities are created in these digital environments and how they work across complex and intersecting ways. In other words, the analytical focus is not simply on the meanings which are transmitted through body pedagogies. Nor is this to focus attention on those engagements with the digital that are simply about resistance. Rather, it means focusing on the relationality between technology, its production and reception as a pedagogical process. The spaces within which this interaction occurs may mean that surveillance and ideological oppression are stronger. As such, even where there are evidenced moments of resistance, these may not be as strong as the powerful forces of surveillance of body pedagogies that may be oppressive, normative or damaging.

\section{Public Pedagogy for Disembodied Cyborgs}

Many approaches to analysing digital health encounters presume a relatively fixed notion of selfhood, the body, and corporeality. Yet, we are at a unique moment in human history where the body is made more complicated by its posthuman technological mediation and prostheticisation. These circumstances are made apparent in the trajectory towards mobile culture, where the mundane cyborg is made manifest in the way that mobile devices become extensions of ourselves and our bodies. Their transformation into wearable technologies like Google Glass and the Oculus Rift, reveal how biopedogogies are mutating around the hybrid body.

To this end, a final dimension of the public pedagogy approach to digital health requires us to consider the embodied nature of pedagogy and how that is changing. As Gaztambide-Fernandez and Arraiz-Matute [49] (p. 57) argue, conceptualising technologies as pedagogical means taking "account of the desires, intentions and conditions that produce them as such". In this vein, understanding how and what people learn about their health in technologically mediated environments means recognising the importance of embodiment in articulations of learning within theories of public pedagogy: 
Public pedagogy articulates this embodied process through which subjectivity is produced and the politics of this process need to be considered across a broader array of spaces, texts and through new theoretical assemblages. Embodiment, therefore, cannot be overlooked in any consideration of the consumption of popular cultural forms [23].

An attempt to define a more embodied and affective understanding of learning is found in Probyn's [54] exploration of materiality affects in the classroom and her focus on "what actual bodies in the classroom do". In turn, this has given rise to discussions about relationalities between bodies as an important aspect of pedagogy, but which have yet to be applied to the context of embodied learning in digital environments. Our intention is not to advance theoretical approaches occupying the "turn to affect" (see [55,56]). Rather, it is to recognize the importance of applying an embodied approach to public pedagogies of technologies alongside the important, but broader, political focus of the dominant discourses of neoliberal regimes of health, the body and citizenship. Approaching digital health in terms of both its biopolitical and embodied nature enables us to engage with critical approaches beyond those operating a broader "levels of abstraction" [54] (p. 25).

Inspired by Deleuzian frameworks, such work focuses on the body in terms of capacities and transformations [57,58] as it is brought into connection with other bodies and technologies [57]. Such conceptualisations are significant insofar as they require us to consider broader ontological complexities and debates about the virtual body, where the body is considered to be both absent and present. The disruption to our bodily continuity that follows from life within mobile digital worlds, requires us to revisit longstanding philosophical and sociological assumptions about what a "body can do" [54,59,60]. Thus, attending to the relational aspects of "inhabiting (digital health) spaces" [49], also means understanding the embodied history that is brought to bear on the affective response one has to new engagements with the body emerging through wearable technologies, visualisation of data etc.:

What constitutes an affective response is hugely complex, and is in part the result of an embodied history to which and with which the body reacts, including how the classroom is conceived and practiced [54] (p. 30).

New sensorial experiences, such as the wearing of fitbit health bands, which vibrate when you achieve your activity goals, combine different pedagogical forces to produce embodied ways of knowing. These new knowledges are not just about techniques of the body [40] (p. 70) or users knowing "how to use their bodies" but also reveal how to use information about their bodies in accordance with the affective responses to that information. In this sense, we can conceptualise digital health as existing at the nexus between discourse, affect and pedagogy and through which subjectivities are constituted as a relationality.

In sum, critical analyses of digital health should engage with "considerations of the affective nature of public, popular and cultural pedagogies" [23] (p. 234). This means a sharper analytic focus on the emotional exchanges occurring in the social media spaces associated with these health apps and the affects emerging through interactions between embodied persons, including those that are constituted as "digital bodies". The inter-corporeality and "trans-subjectivity" [56] of bodies, means we cannot think of their presence in digital spaces as singular and bounded. They are connected to other bodies through digital platforms and with technologies; increasingly so as we move towards web 3.0 and the 
Internet of Things. Thus, the prostheticised body occupies spaces of ambiguity where it is always unfinished and always compelled to improve its digital rendering.

The affective flows of pedagogy in these digital spaces can operate as both power and pleasure. The Web 2.0 era marks a shift towards health platforms that are oriented around what might be described as "playful" environments. The mashing up of the quantification of self through the collection of data about oneself, with creative and game play scenarios, is a recent trend described by Whitson [35] as "gamification". This is notable for the way in which "playful frames" are "applied to non-play spaces" [35] (p. 166). In this way, digital health pedagogies may be experienced as "play" $[35,61,62]$. Many contemporary health and fitness apps draw on gamification whereby "playful frames" are "applied to non-play spaces" such that everyday tasks, such as running, become more enjoyable or more like a game. Zombie Run is a prominent example of this trend; an app which combines social media technology with game play situations featuring zombies, as stated on its website "Join 800,000+ runners on an epic adventure that motivates you to run further and faster than ever before-whether you're a beginner or an expert!". To this extent, such explanatory concepts reveal how applications engaging with gamification can neither be reduced to being simply oppressive, nor emancipatory. On the one hand, Zombie Run exhorts individuals to undertake physical activity and monitor and regulate one's lifestyle. At the same time, users might experience more pleasurable aspects of physical activity, such as exploration of local space through new running routes via the zombie play feature or new routes suggested by running apps. For a participant of these environments, it may be possible to experience both, at the same time. Other examples include health apps that use rewards for healthy eating habits. So, through pedagogical processes like gamification, digital health technologies are normative, regulatory and resistant. Digital health may therefore present new frames of engagement which can be both enabling and constraining at the same time. In this sense, digital environments present us with perhaps, multiple and often contradictory pedagogical forces.

These examples situate health apps in the complex body-machine assemblages through which bodies enter into complex inter-corporeal affectivity, experiencing pleasure, shame, disgust, enjoyment. Probyn [54] (p. 26) describes how "affect amplification makes us care about things". Many health apps monitor users movements and bodily functions and require users to upload data wirelessly. Many users have the opportunity to share their data in social media environments, sharing data or discussing behaviours with others online. The linking of bodies in these spaces is indicative of bodies entering into entangled processes, and the affective capacities, even where the corporeal body might not be present. In this sense, rather than simply being shaped by social influences, bodies entering into these digital assemblages are part of the co-constitutive process. These inter-subjective encounters are a crucial component of public pedagogy, where "subjects come into relationship and influence each other" [31,49]. Perhaps crucially, one of the questions that arises from this is "whose desires and intentions are enacted and imposed pedagogically and to what end...who is it we want people to become" [49] (p. 59).

\section{Conclusions}

This paper has outlined a critical approach to understanding digital health in the context of theories of public pedagogy. It began by arguing on behalf of a public pedagogy approach, before setting out 
some of the formal parameters of this that are most suited to an analysis of digital health encounters. In so doing, we have outlined differences within public pedagogy theory and highlighted how "theories of "pedagogy writ large" have the potential to provide diverse traditions with a mediating concept between the production of knowledge or ideology and the normative power it exercises" [23] (p. 234). We went on to outline how public pedagogy approaches draw attention to the neoliberal governance of the body, which occurs through digital health encounters. However, we also acknowledge the limitation of this broad vision of public pedagogy, which can obfuscate the various ways in which multiple pedagogic forces intersect, which is particularly prominent within virtual worlds. As such, we present a conceptual framework for public pedagogy that takes into account the limitations of a focus on digitality as popular culture at the broadest level. This approach emphasises more complex processes that operate around crucial categorical distinctions, such as between the virtual and non-virtual, the individual and the community, public and private, and formal or informal educational structures. We have also acknowledge the changing conditions of embodiment that arise from a trajectory towards greater digitalization, where "online bodies are bodies that are certainly being written, but simultaneously bodies to write on" [63] (p. 229). In the digital apps we describe, users are not merely the "machine parts" [63] (p. 219) of the human-computer interface, but are made "more autonomous" [63] through their "symbiotic relationship to machines" [63].

There is a lack of research on individual's experiences of these technologies, particular in terms of the implications for "embodiment, selfhood and social relationships" [5] (p. 299). The intention here has been to recognize the importance of applying an embodied nature of pedagogy alongside broader political focus of public pedagogy [34] to better understand how digital health encounters take place. Making the body more central to conceptualizations of public pedagogies of technology, while attending to the political and affective dimensions of technological relationships, ensures a more critical understanding of what occurs in digital health, since it does not neglect how the relational dimensions of networked experiences brings an additional pedagogic layer, beyond the content. This approach provides a useful way of negotiating the polarisations between utopian and dystopian views of digital health, but it does not ignore or diminish the aspirations or anxieties that arise from these views.

\section{Acknowledgments}

The authors would like to acknowledge the support of the University of Bath and the University of the West of Scotland.

\section{Author Contributions}

This article was co-devised and co-authored by Emma Rich and Andy Miah. All authors read and approved the final manuscript.

\section{Conflicts of Interest}

The authors declare no conflict of interest. 


\section{References}

1. Bell, D.; Kennedy, B. The Cybercultures Reader; Routledge: London, UK and New York, NY, USA, 2000.

2. Gauntlett, D.; Horsley, R. Web.Studies; 2nd ed.; Arnold Publishers: London, UK, 2004.

3. Haraway, D.J. A Cyborg Manifesto: Science, Technology, and Socialist-Feminism in the Late Twentieth Century; Routledge: New York, NY, USA, 1991.

4. Stelarc. From Psycho to Cyber Strategies: Prosthetics, Robotics and Remote Existence. Cult. Values 1997, 1, 241-249.

5. Lupton, D. M-health and health promotion: The digital cyborg and surveillance society. Soc. Theory Heal. 2012, 10, 229-244.

6. Bauer, K.A. Home-Based Telemedicine: A Survey of Ethical Issus. Cambridge Q. Healthc. Ethics 2001, 10, 137-146.

7. Collste, G. The Internet Doctor and Medical Ethics: Ethical implications of the introduction of the Internet into medical encounters. Med. Heal. Care Philos. 2002, 5, 121-125.

8. Baym, N.K. Personal Connections in the Digital Age; Polity: Cambridge, UK, 2010.

9. Byron, T. Safer Children in a Digital World: The Report of the Byron Review. Available online: http://webarchive.nationalarchives.gov.uk/20130401151715/http://www.education.gov.uk/publica tions/eOrderingDownload/DCSF-00334-2008.pdf (accessed on 22 April 2014).

10. Greenfield, S. Computers may be altering our brains. The Independent, 12 August 2011. Available online: http://www.independent.co.uk/voices/commentators/susan-greenfield-computers-may-bealtering-our-brains-2336059.html (accessed on 21 May 2014).

11. Hine, C. Virtual Ethnography; Sage: London, UK, 2000.

12. Rheingold, H. Virtual Reality; Manderin: London, UK, 1991.

13. Turkle, S. Life on the Screen: Identity in the Age of the Internet; Weidenfeld and Nicolson: London, UK, 1995.

14. Arthur, C. Apps more popular than the mobile web, data shows. The Guardian, 2 April 2014. Available online: http://www.theguardian.com/technology/appsblog/2014/apr/02/apps-more-popularthan-the-mobile-web-data-shows (accessed on 21 May 2014).

15. Fox, S.; Duggan, M. Tracking for Health. Available online: http://www.pewinternet.org/2013/01/28/ tracking-for-health/ (accessed on 21 May 2014).

16. Mager, A. Search engines matter: From educating users towards engaging with online health information practices. Policy Internet 2012, 4, 1-21.

17. Chan, M. Mobile phones and the good life: Examining the relationships among mobile use, social capital and subjective well-being. New Media Soc. 2013, doi:10.1177/1461444813516836.

18. Miah, A.; Rich, E. Medicalization of Cyberspace; Routeldge: London, UK and New York, NY, USA, 2008.

19. Nettleton, S.; Burrows The Emergence of E-Scaped Medicine? Sociology 2004, 38, 661-679.

20. Sandlin, J.A.; Malley, M.P.O.; Burdick, J. Mapping the Complexity of Public Pedagogy Scholarship: 1894-2010. Rev. Educ. Res. 2011, 81, 338-375.

21. Andrews, D.L. Kinesiology's Inconvenient Truth and the Physical Cultural Studies Imperative. Cult. Stud. 2008, 60, 45-62. 
22. Freishtat, R.L.; Sandlin, J.A. Shaping Youth Discourse about Technology: Technological Colonization, Manifest Destiny, and the Frontier Myth in Facebook's Public Pedagogy. Educ. Stud. 2010, 46, 503-523.

23. Hickey-Moody, A.; Savage, G.C.; Windle, J. Pedagogy writ large: Public, popular and cultural pedagogies in motion. Crit. Stud. Educ. 2010, 51, 227-236.

24. Ruckenstein, M. Visualized and Interacted Life: Personal Analytics and Engagements with Data Doubles. Societies 2014, 2, 68-84.

25. Bauman, Z. Consuming Life; Polity: Cambridge, UK, 2007.

26. Burdick, J., Sandlin, J., O’Malley, M.P., Eds. Problematizing Public Pedagogy; Routledge: New York, NY, USA, 2014.

27. Giroux, H.A. Public Pedagogy and the Politics of Neo-liberalism: making the political more pedagogical. Policy Futur. Educ. 2004, 2, 494-503.

28. Giroux, H.A.; Hall, S.; Grossberg, L.; Kellner, D.; Morris, M.; Miller, T. Cultural Studies, Public Pedagogy, and the Responsibility of Intellectuals. Commun. Crit. Stud. 2004, 1, 59-79.

29. Kellner, D. Media Culture: Cultural Studies, Identity and Politics between the Modern and the Postmodern; Routledge: New York, NY, USA, 1995.

30. Ellsworth, E. Places of Learning: Media, Architecture, and Pedagogy; Routledge: New York, NY, USA, 2005.

31. Sandlin, J.A.; Milam, J.L. Mixing pop (culture) and politics: Cultural resistance, culture jamming and anti-consumption activism as critical public pedagogy. Curric. Inq. 2008, 38, 323-350.

32. Sandlin, J.; Schultz, B.; Burdick, J. Handbook of Public Pedagogy: Education and Learning Beyond Schooling; Routledge: New York, NY, USA, 2010.

33. Savage, G. Problematizing "public pedagogy" in educational research. In Handbook of Public Pedagogy; Sandlin, A.J., Schultz, B.D., Burdick, J., Eds.; Routledge: New York, NY, USA, 2010; pp. 103-115.

34. Giroux, H.A. Border Crossings: Cultural Workers and the Politics of Education; Routledge: New York, NY, USA, 1992.

35. Whitson, J. Gaming the Quantified Self. Surveill. Soc. 2013, 11, 163-176.

36. Swan, M. Health 2050: The Realization of Personalized Medicine through Crowdsourcing, the Quantified Self, and the Participatory Biocitizen. J. Pers. Med. 2012, 2, 93-118.

37. Giroux, H. Public Pedagogy as Cultural Politics: Stuart Hall and the "Crisis" of Culture. Cult. Stud. 2000, 14, 341-360.

38. Giroux, H.A. Cultural Studies in Dark Times: Public Pedagogy and the Challenge of Neoliberalism. Available online: http://www.henryagiroux.com/online_articles/DarkTimes.htm (accessed on 21 May 2014).

39. Foucault, M. The History of Sexuality, Volume I: An Introduction; Penguin Books: Harmondsworth, Middlesex, UK, 1976.

40. Mauss, M. The Techniques of the Body. Econ. Soc. 1973, 2, 70-88.

41. Bell, K. App Store Pulls "Barbie" Plastic-Surgery App Following Backlash. Available online: http://mashable.com/2014/01/14/barbie-plastic-surgery-app/ (accessed on 24 February 2014).

42. Evans, J.; Rich, E.; Davies, B.; Allwood, R. Education, Disordered Eating and Obesity Discourse: Fat Fabrications; Roultedge: Abingdon, UK, 2008. 
43. Douglas, M. Purity and Danger; Roultedge: London, UK, 1966.

44. Shilling, C. Afterword: Embodiment, Social Order, and the Classification of Humans as Waste. Societies 2013, 3, 261-265.

45. Beer, D.; Burrows, R. Consumption, Prosumption and Participatory Web Cultures: An introduction. J. Consum. Cult. 2010, 10, 3-12.

46. Van Dijck, J. Users like you? Theorizing agency in user-generated content. Media Cult. Soc. 2009, 31, 41-58. Available online: http://jclass.umd.edu/classes/jour698m/vandijk.pdf (accessed on 3 June 2014).

47. Evans, J.; Rich, E.; Holroyd, R. Disordered Eating and Disordered Schooling: What Schools Do To Middle Class Girls. Br. J. Sociol. Educ. 2004, 25, 123-142.

48. Livingstone, S.; Haddon, L. EU Kids Online: Final Report. Available online: www.lse.ac.uk/ EUKidsOnlineFinalReport (accessed on 3 June 2014).

49. Gaztambide-Fernández, R.; Arraiz-Matute, A. "Pushing Against": Relationality, Intentionality, and the Ethical Imperative of Pedagogy. In Problematizing Public Pedagogy; Burdick, J., Sandlin, J.A., O’Malley, M.P., Eds.; Routeldge: New York, NY, USA; pp. 52-64.

50. Wright, J.; Harwood, V. Biopolitics and the Obesity Epidemic: Governing Bodies; Routledge: London, UK, 2009.

51. Walkerdine, V. Biopedagogies and Beyond. In Biopolitics and the Obesity Epidemic: Governing Bodies; Wright, J, Harwood, V, Eds.; Routledge: New York, NY, USA and Oxon, UK, 2009; pp. 199-207.

52. Fullagar, S. Governing health family lifestyles through discourses of risk and responsibility. In Biopolitics and the Obesity Epidemic: Governing Bodies; Wright, J., Harwood, V., Eds.; Routledge: New York, NY, USA, 2009; pp. 108-126.

53. Savage, G.C. Chasing the Phantoms of Public Pedagogy: Political, Popular and Concrete Publics. In Problematizing Public Pedgagogy; Burdick, J., Sandlin, J.A., O’Malley, M.P., Eds.; Routledge: New York, NY, USA, 2014; pp. 79-90.

54. Probyn, E. Teaching Bodies: Affects in the Classroom. Body Soc. 2004, 10, 21-43.

55. Massumi, B. Parables for the Virtual: Movements, Affect, Sensation; Duke University Press: Durham, NC, USA and London, UK, 2002.

56. Blackman, L.; Featherstone, M. Editorial. Body Soc. 2009, 15, 1-2.

57. Grosz, E. Volatile Bodies: Toward a Corporeal Feminism; Indiana University Press: Indianapolis, IN, USA, 1994; Volume 5.

58. Coleman, R. The becoming of bodies: Girls, media effects and body image. Fem. Media Stud. 2008, 8, 163-179.

59. Deleuze, G. Ethology: Spinoza and Us. In Incorporations; Lotringer, S., Ed.; Zone: New York, NY, USA, 1992.

60. Latour, B. How to Talk About the Body? The Normative Dimensions of Science Studies. Body Soc. 2004, 10, 205-229.

61. Ellerbrok, A. Playful Biometrics: Controversial Technology through the Lens of Play. Sociol. $Q$. 2011, 52, 528-547.

62. McGrath, J.E. Loving Big Brother: Performance, Privacy and Surveillance Space; Routledge: London, UK, 2004. 
63. Sundén, J. What Happened to Difference in Cyberspace? The (Re)Turn of the She-Cyborg. Fem. Media Stud. 2001, 1, 215-232.

(C) 2014 by the authors; licensee MDPI, Basel, Switzerland. This article is an open access article distributed under the terms and conditions of the Creative Commons Attribution license (http://creativecommons.org/licenses/by/3.0/). 